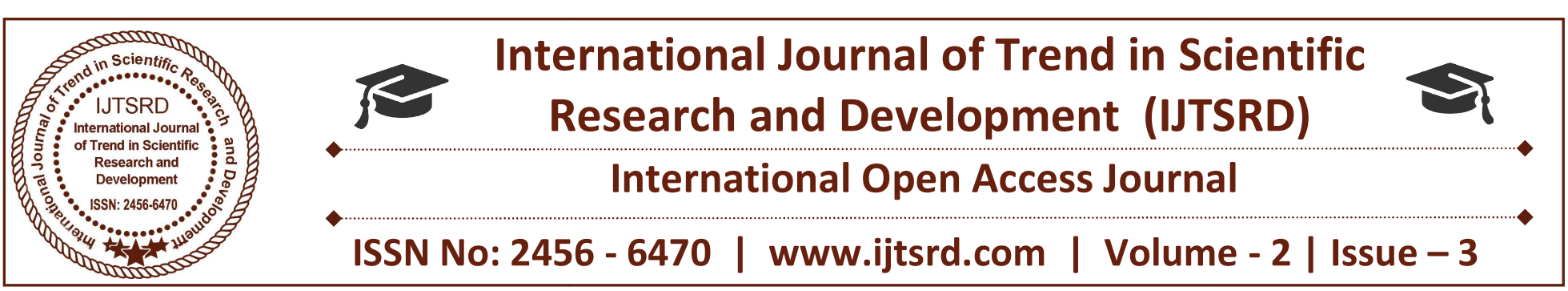

\title{
Investigation of Urinary Tract Disease : A Short Review
}

\author{
${ }^{1}$ Kumar Rajeev, ${ }^{2}$ Rathore Garima, ${ }^{3}$ Gayatri \\ ${ }^{1}$ Assistant Professor, ${ }^{2,3}$ P.G. Scholar \\ Department of Shalya Tantra Patanjali Bhartiya Ayurvigyan Evam \\ Anusandhan Sansthan, Haridwar, Uttarakhand, India
}

\begin{abstract}
Urology is one of the most competitive and highly sought surgical specialties for surgeons. There are many urological conditions like $\mathrm{BPH}$, Carcinoma prostate, Bladder tumour, Urinary incontinence, Urinary tract infection, Urolithiasis etc. An actual diagnosis is very important issue to treat these conditions. In modern era investigations are essential part to make a diagnosis. Without investigations diagnosis is uncertain. In urology there are many investigations like Urine, KFT, Intravenous pylography, retrograde urethrogram, Angiography, Ultrasonography etc. In this article a short and essential view of some urological investigations are given.
\end{abstract}

\section{Keywords: Urology, Investigation, Urolithiasis}

\section{INTRODUCTION}

Urology is rapidly becoming superspeciality branch in present scenario . In present population urological condition such as prostate cancer, urinary incontinence, and lower urinary tract conditions like stricture are becoming more prevalent .

Prevalence of urinary tract disease is more in children and female. In old age males usually after 40 are more prone to urinary tract disease like Prostatomegaly Prostate cancer, urine incontinence etc. In modern era with increasing incidence of disease like Diabetes Mellitus, hypertension, sexually transmitted disease , improper use of medicine like antibiotics, analgesics etc, injustified use of pesticides, insectides have raised the incidence of urinary tract disease .

Congenital malformation of urinary tract are not very less common .Sooner or later the symptoms present in the patient either immediately after birth, in infancy , childhood or at late age. Congenital malformation / abnormality of urinary tract are readily being detected by pediatricians or pediatric urologist or urologist with the recent advances of investigations.

With the daily advancement in research in investigation it has become easier and helps in making correct diagnosis. The investigations can be basic like specific gravity, $\mathrm{pH}$, urine protein etc whereas when complicated special investigations like urogram, cystography or radioactive isotopes like DTPA ,TcDM etc .

They are classified as following:

\section{URINE EXAMINATION ${ }^{1}$ :}

\section{Physical}

Colour: normal colour of urine is amber colour and clear. It may vary from light amber to yellow colour depending on the concentration of urine . Pathological changes occur due to the presence of bile pigments ,urobilinogen ,pus, blood ,crystals. Several drugs also may change the colour of urine.

Volume: the total amount of urine excreted in $24 \mathrm{hrs}$ .The time of collection along with volume collected should be charted. Usually collection time starts from $8 \mathrm{AM}$ to next $24 \mathrm{hrsin}$ a bottle containing a preservative usually $10 \mathrm{ml}$ of glacial acetic acid. Normal value is 700 to $2500 \mathrm{ml}$. its collected for 
special test like estimation of 17 ketosteroids, VMA, or other metabolites .

Specific gravity: This is measured using a urinometer. The specific gravity depends upon the concentration of solutes present in urine. Crystalloids like salts sugar and urea raise specific gravity it varies from 1.005 to 1.040 .

Osmolality: its more accurate parameter to express the concentration of solutes in urine it can be measured using an osmometer. Osmolality of urine varies from 50 to $1200 \mathrm{mOsm} / \mathrm{kg}$ water. Osmolality and specific gravity indicates the concentrating ability of the kidney.

$\mathbf{p H}$ : The urinary $\mathrm{pH}$ normally ranges from 4.5 to 8 . $\mathrm{pH}$ is tested with litmus paper. It varies depending on serum $\mathrm{pH}$. Urine $\mathrm{pH}$ influences the type of stone formed .Uric acid stones occur in acidic $\mathrm{pH}$ whereas in alkaline urine secondary stones develop. Conditions like $\mathrm{E}$ coli infection makes urine strongly acidic whereas infection by B.proteus makes the urine strongly alkaline.

\section{Chemical}

Protein : Normal urine contain minute amount of protein which is too low to be detected by routine test. Now it is defined as excretion of protein more than $150 \mathrm{mg} /$ day. It occurs in condition like glomerulonephritis and nephrotic syndrome.

1) Heat cogulation and acetic acid test: On heating urine opacity developes due to cogulated protein.

2) Dipstix test: Filter paper strip impregnated with chemicals are used .These sticks change colour in presence of sugar, protein and nitrites.

3) Radioimmunoassay: Microalbuminuria

4) Bence-jones protein: This consist of light chains of abnormal protein secreted by neoplastic cells in multiple myeloma and other cell dyscrasis. It is also a heat test.

Sugar : Sugar is detected by boiling urine with Fehling's reagent or Benedict 's reagent. Specific test for glucose is glucose oxidase test. Glucostix test strips are available commercially. Test strips are unaffected by other sugars and drugs. It is more reliable.
Acetone: Rothera test is performed to check the presence of ketone bodies in urine . Ketone body appears in diabetic ketoacidosis, Prolonged starvation and repeated vomiting.

\section{BIOCHEMICCAL TESTS ${ }^{2,3}$ :}

Blood urea: It is universally accepted reliable test for renal excretory function. Normal level of blood urea is $20-40 \mathrm{mg} / \mathrm{dl}$.

Serum Creatinine: Normal value is $.7-1.2 \mathrm{mg} / \mathrm{dl}$. Creatinine is filtered completely by glomeruli and is not significantly reabsorbed or secreted by renal tubule .Level of serum creatinine thus remains steady over prolonged periods. Hence Creatinine clearance is reliable clinical parameter of glomerular filtration rate.

Serum uric acid: Uric acid is the end product of purine metabolism and is excreated by kidney.

Normal level: In males- 2.5 to $8 \mathrm{mg} / \mathrm{dl}$ In females- 1.5 to $6 \mathrm{mg} / \mathrm{dl}$

Serum electrolyte : Serum electrolyte chiefly sodium ,potassium and bicarbonates is severly affected by deranged kidney function hence they have to be closely watched during renal failure .Calcium phosphorus are also deranged in chronic renal failure.

Prostate surface antigen (PSA) ${ }^{\mathbf{5}}$ :This is the most important tumour marker now available .It is a glycoprotein elevated in prostatic diseases. Its estimation is used for screening of carcinoma prostate but it is not specific for this condition. Normal level is $0-4 \mathrm{ng} / \mathrm{ml}$. If PSA is more than $4 \mathrm{ng} / \mathrm{ml}$ carcinoma is suspected.

\section{RADIOLOGY ${ }^{1,2,3,4}$}

\section{Plane radiograph of abdomen (KUB X-RAY).}

A proper taken KUB should include abdomen from $11^{\text {th }}$ thoracic vertebrae to lower margin of inferior pubic remi and both flank lines of extra peritoneal fats .It is done to find out the presence of calculi in urinary passage ,soft tissue calcification specially in renal parenchyma and arteries and abnormalities in vertebrae and pelvic bones .

Intravenous pyelography and Intravenous urogram : It is useful for assessing renal function as well the anatomy of urinary passage. In this procedure bowel preparation is done 2-3 days prior to avoid gas 
shadow .The patient should not take oral fluid 6 hours prior to procedure. The dye used is $45 \%$ sodium diatrizoate . 20-40 $\mathrm{ml}$ of this dye is injected to median cubital vein .Now the films are taken at a interval of

1) Early radiograph after 2 to 5 minutes- Nephrogrm

2) After 5 minutes -Pyelogram

3) After 15 to 20 minutes -Ureter and bladder is visualized

4) Once the bladder is visualized post voiding picture is taken to show any residual contrast in urinary bladder.

Retrograde pyelogram :The urinary passage is visualized by introducing contrast dye into the ureter by retrograde catheterization through a cystoscope. Uretheric catheter are introduced and 4to $6 \mathrm{ml}$ of iodinated dye is injected into the ureter or renal pelvis and pictures are taken. This brings out pelvicalyceal system and ureter clearly.

Antegrade pyelogram: The radio contrast dye is injected directly into the renal pelvis through percutaneous puncture or nephrostomy tube. Micturating cystourography is taken.

This help to identify:

Reflux of urine from the bladder to one or poth ureter

2) To detect posterior

3) Vesical

urethral wall

4) Vesicovaginal fistula

\section{Angiography}

Flush abdominal aortogram : This study is necessary to visualized the vascular pattern of the kidney of donor for transplantation .

Selective renal arteriogram : A special arteriography catheter is positioned in renal artery and pictures are taken after dye injection. This help to study arterial pattern of kidney in detail in addition dilation of narrow segments using balloon, occlusion of vessels of tumors of vascular malformation .

Digital substraction angiogram : This gives an angiographic picture without interference from other structures like bones, gas and soft tissue hence its clarity is very good.

\section{USG- (Ultrasonography)}

It is a non-invasive investigation modality in the diagnosis of anatomical abnormalities of urinary system .It can be used through different approaches transabdominal, transrectal, transvaginal .It helps to visualise kidney, adrenals ,ureter ,urinary bladder and prostate ,their size ,morphological abnormalities like enlargement, contraction, distortion, tumour, cyst , abscess, stones etc.USG should be performed before undertaking any invasive procedure like IVU.

\section{Computed Tomography Scan}

It reveals the size ,morphology,vasculature normalities and abnormalities .It is helpful in detection and staging of malignancies of urinary tract.

CT urogram, MR angio, spiral CT and MRI scan are other recent diagnostic investigation.

\section{ENDOSCOPY :}

Cystoscopy- Bladder can be visualized .It helps in therapeutics procedure such as removal of stone and biopsy.

\section{HISTOPATHOLOGICAL STUDY}

Percutaneous renal biopsy - It is an invasive procedure. It is the most specific diagnostic method in renal disease .It is of great value in estabilising the pathology. It is done with automatic spring loaded biopsy gun under USG guidance .

\section{Isotopic studies :}

DTPA (Diethylene triamine penta-acetic acid)-It is employed to study renal blood flow, Obstructed uropathy ,bladder empting and glomerular filtration rate .It is also known as Dynamic Renal Scintigraphy .

DMSA (Dimercapto succinic acid )-It is most commonly used for cortical imaging. It shows detail of renal parenchyma .It is particularly useful when looking for segmental abnormalities of kidney like renal scarring .

MR (Magnetic resonance) Urography : MRI of genito urinary system in many situation like 1) For accurate evaluation of IVC thrombus in renal cell carcinoma .

2) It is also

useful for delination of uretheral injuries.

In this article its here by updated all latest investigations like MRI ,scintigraphy histopathology 
etc along with the old and basic investigation .urinary tract has same clinical presentation but the pathology is different the advancement of the investigations has helped all clinicians along especially urologist to make proper diagnosis and treat the patient .

\section{REFERENCES}

1. Das Krishna KV .Clinical Medicine A text book of Clinical Methods and Laboratory Investigations. Fourth edition Viswesaran Kasi R Chapter 30 Investigations in Nephrology p.354368.
2. Shenoy Rajgopal K and Shenoy Anitha . Manipal manual of surgery. Fourth edition Chapter 38 Investigations of the urinary tract. p. 923-931.

3. Das.S A Concise Text Book of Surgery Ninth edition .Chapter 48 The kidney and ureter p.11721179.

4. Das.S A Concise Text Book of Surgery Ninth edition, Chapter 49 The urinary bladder, p.1248

5. Das.S A Concise Text Book of Surgery Ninth edition, Chapter 50 The Prostate and urethra p.1286-1288

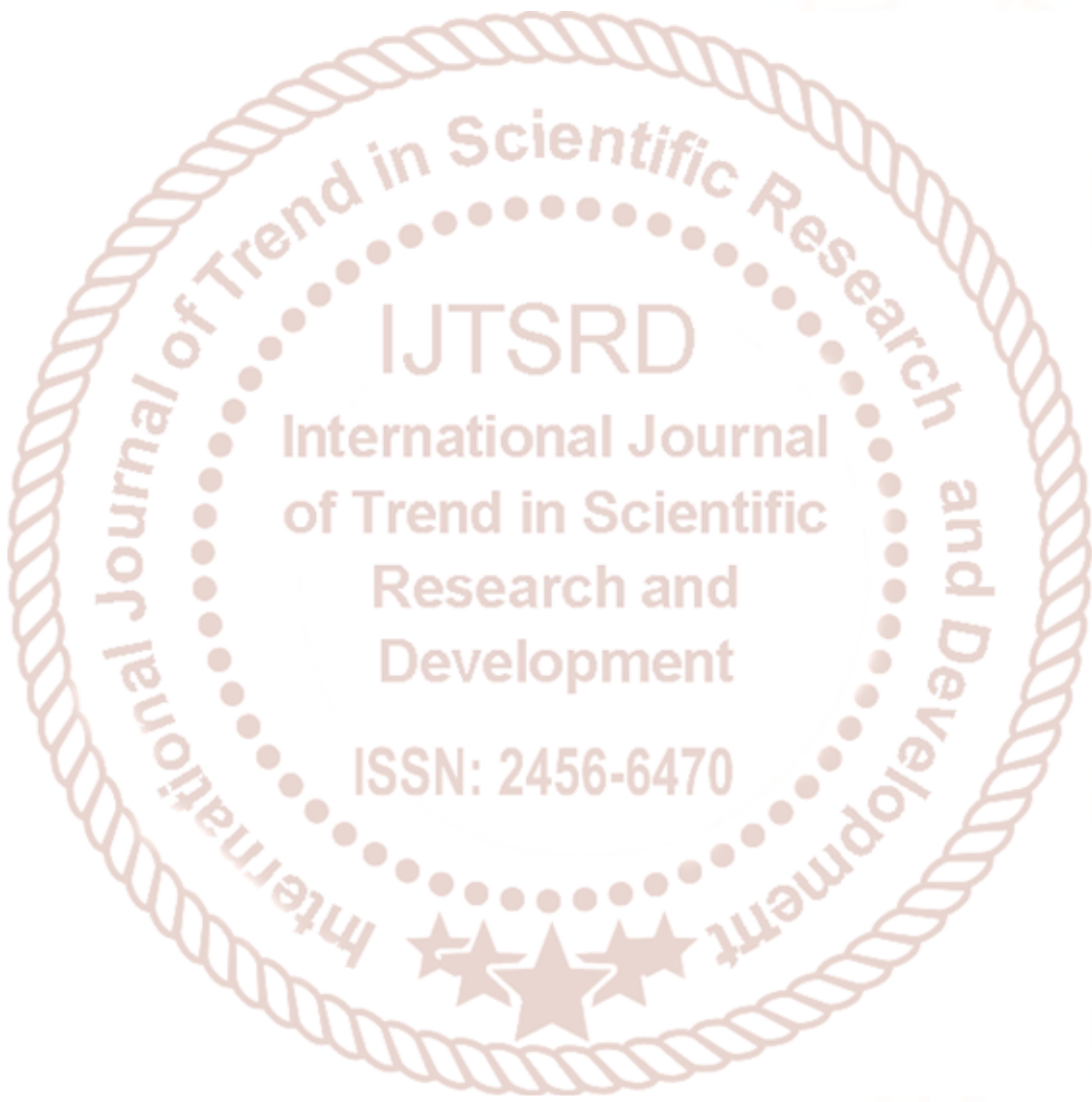

